\title{
Iterated function systems consisting of F-contractions
}

Nicolae-Adrian Secelean*

\begin{abstract}
In this paper we consider a particular case of a contractive self-mapping on a complete metric space, namely the F-contraction introduced by Wardowski (Fixed Point Theory Appl. 87, 2012, doi:10.1186/1687-1812-2012-94), and provide some new properties of it. As an application, we investigate the iterated function systems (IFS) composed of $F$-contractions extending some fixed point results from the classical Hutchinson-Barnsley theory of IFS consisting of Banach contractions. Some illustrative examples are given.
\end{abstract}

MSC: Primary 28A80; secondary 47H10; 54E50

Keywords: contractive map; F-contraction; iterated function system; attractor; Hausdorff-Pompeiu metric

\section{Introduction}

One of the basic concepts of fractals theory is undubitably the iterated function system (IFS) introduced in 1981 by Hutchinson [1] and popularized by Barnsley [2], IFS being the main generator of fractals. This consists of a finite set of contractions $\left(\omega_{k}\right)_{k=1}^{K}$ on a complete metric space $X$ into itself. For such an IFS, there is anyway a unique nonempty compact set $A \subset X$ such that $A=\bigcup_{k=1}^{K} \omega_{k}(A) . A$ is, generally, a fractal set and it is called the attractor of the respective IFS. During the last decades, many authors have been concerned with the extension of this framework to more general spaces, generalized contractions and infinite IFSs or, more generally, to multifunction systems.

Miculescu and Mihail introduced in [3, 4] the generalized iterated function system (GIFS) consisting of a collection of contractions (respectively, contractive functions) on $X^{I}=\left\{\left(x_{i}\right)_{i \in I} ; x_{i} \in X\right\}$ endowed with the maximum metric into $X$, when $X$ is a complete (respectively compact) metric space and $I$ is finite. Dumitru in [5] and, respectively, Strobin and Swaczyna in [6] improved the work of Miculescu and Mihail by considering GIFSs composed of Meir-Keeler type mappings and, respectively, $\varphi$-contractions. Secelean [7] investigated the iterated function systems composed of a countable family of contractive, respectively $\varphi$-contractions, Meir-Keeler type maps. Some remarkable results concerning extensions of the metric spaces, on which the IFSs are defined, and generalizations of their contractions can be found, for example, in $[8,9]$ and others.

The famous Banach-Picard-Caccioppoli theorem (also called the contraction principle) states that, given a complete metric space $(X, \mathrm{~d})$ and a function $\omega: X \rightarrow X$ for which there

@2013 Secelean; licensee Springer. This is an Open Access article distributed under the terms of the Creative Commons Attribution License (http://creativecommons.org/licenses/by/2.0), which permits unrestricted use, distribution, and reproduction in any medium, provided the original work is properly cited. 
is $r \in(0,1)$ such that

$$
\mathrm{d}(\omega(x), \omega(y)) \leq r \mathrm{~d}(x, y), \quad \forall x, y \in X,
$$

there exists a unique $e \in X$ such that $\omega(e)=e$. Furthermore, for every $x \in X$, the sequence $\left(\omega^{p}(x)\right)_{p}$ converges to $e$, where $\omega^{p}=\omega \circ \omega^{p-1}(p$ time composition of $\omega), p \geq 1$. The map $\omega$ which satisfies the previous condition is called $r$-contraction or, more, Banach contraction. Also, in the above setting, we say that $e$ is the fixed point of $\omega$.

Many authors have provided several extensions of this result. In this respect, they considered the contractive mappings, namely those functions $\omega: X \rightarrow X$ that satisfy the inequality

$$
\mathrm{d}(\omega(x), \omega(y))<\mathrm{d}(x, y), \quad \forall x, y \in X, x \neq y .
$$

Niemytzki-Edelstein's theorem (see, e.g., [10, Th. 2.2, p.34]) states that each contractive mapping on a compact metric space into itself has a unique fixed point which is successively approximated by the iterates of the respective map at every point of the space. Some other particular cases in which the considered metric space is complete are investigated in fixed point theory. In this regard, starting from a function $F:(0, \infty) \rightarrow \mathbb{R}$ satisfying some suitable properties, Wardowski provided in [11] a new type of such a contractive mapping, namely $F$-contraction, and proved a fixed point theorem concerning $F$-contractions on a complete metric space. Further, an example of a map which is an $F$-contraction while it is not a Banach contraction was given.

In this paper, we simplify one of the required conditions for $F$ (Remark 3.1) and describe a large class of such functions $F$ (Proposition 3.1, Remark 3.2 and Remark 3.3). Next, we consider an iterated function system (IFS) composed of $F$-contractions and prove that it always has a unique attractor (Theorem 4.1). We further provide some conditions in which the limit of a sequence of IFSs is also an IFS and its attractor is the limit of the sequence of the corresponding attractors (Theorem 4.2). A significant example in this respect is given. Notice that all the mappings which compose the IFSs from that example are not Banach contractions.

Throughout the whole paper, by $\mathbb{R}$ we understand the set of real numbers and by $\mathbb{R}_{+}$we denote the set of all positive real numbers $(0, \infty)$. The set of all positive integers will be denoted by $\mathbb{N}$.

\section{Preliminary facts: Hausdorff-Pompeiu metric}

Let $(X, \mathrm{~d})$ be a metric space and $\mathcal{K}(X)$ be the class of all nonempty compact subsets of $X$. The function $h: \mathcal{K}(X) \times \mathcal{K}(X) \rightarrow[0, \infty), h(A, B)=\max \{\mathrm{D}(A, B), \mathrm{D}(B, A)\}$, where $\mathrm{D}(A, B)=$ $\sup _{x \in A} \inf _{y \in B} \mathrm{~d}(x, y)$ for all $A, B \in \mathcal{K}(X)$, is a metric called the Hausdorff-Pompeiu metric.

The metric space $(\mathcal{K}(X), h)$ is complete provided that $(X, \mathrm{~d})$ is complete.

Some simple standard facts, which will be used in the sequel, are described in the following lemmas (for details, see, e.g., [12, \$1.4.]).

Lemma 2.1 [12, Prop. 1.1] Let $A, B, C \in \mathcal{K}(X)$. Then

( $\alpha) A \subset B \Leftrightarrow \mathrm{D}(A, B)=0$,

( $\beta) \mathrm{D}(A, C) \leq \mathrm{D}(A, B)+\mathrm{D}(B, C)$. 
Lemma $2.2\left[12\right.$, Th. 1.13] If $\left(E_{i}\right)_{i \in \mathfrak{\Im}},\left(F_{i}\right)_{i \in \mathfrak{\Im}}$ are two finite collections of sets in $(\mathcal{K}(X), h)$, then

$$
h\left(\bigcup_{i \in \mathfrak{\Im}} E_{i}, \bigcup_{i \in \mathfrak{I}} F_{i}\right) \leq \sup _{i \in \mathfrak{\Im}} h\left(E_{i}, F_{i}\right) .
$$

Lemma 2.3 [12, Th. 1.14] Let $\left(A_{n}\right)_{n}$ be a sequence of nonempty compact subsets of $X$.

(a) If $A_{n} \subset A_{n+1}$ for all $n \geq 1$, and the set $A:=\bigcup_{n \geq 1} A_{n}$ is relatively compact, then $\lim _{n} A_{n}=\bar{A}$.

(b) If $A_{n+1} \subset A_{n}$ for every $n \geq 1$, then $\lim _{n} A_{n}=\bigcap_{n \geq 1} A_{n}$,

the limit being taken in the Hausdorff-Pompeiu metric and the bar means the closure.

Throughout this paper, by $\mathcal{K}(X)$ we understand the metric space $(\mathcal{K}(X), h)$.

\section{F-contractions}

We describe here a new type of contractive mappings, namely $F$-contractions, defined by Wardowski in [11] and add some results about them.

Throughout this section, $(X, \mathrm{~d})$ denotes a metric space.

Definition 3.1 [11] Let $F: \mathbb{R}_{+} \rightarrow \mathbb{R}$ be a mapping satisfying:

(F1) $F$ is strictly increasing, i.e., for all $t, s \in \mathbb{R}_{+}, t<s$, one has $F(t)<F(s)$,

(F2) for each sequence $\left(t_{k}\right)_{k}$ of positive numbers, $\lim _{k} t_{k}=0$ if and only if $\lim _{k} F\left(t_{k}\right)=-\infty$,

(F3) there exists $\lambda \in(0,1)$ such that $\lim _{t \backslash 0} t^{\lambda} F(t)=0$.

A mapping $\omega: X \rightarrow X$ is said to be an $F$-contraction if there is $\tau>0$ such that

$$
\tau+F(\mathrm{~d}(\omega(x), \omega(y))) \leq F(\mathrm{~d}(x, y)), \quad \forall x, y \in X, \omega(x) \neq \omega(y) .
$$

We denote by $\mathcal{F}$ the family of all $F: \mathbb{R}_{+} \rightarrow \mathbb{R}$ which satisfy conditions (F1), (F2) and (F3).

We will show that condition (F2) can be replaced by an equivalent but a more simple one. First, we need to prove the following two elementary lemmas.

Lemma 3.1 If $\left(t_{k}\right)_{k}$ is a bounded sequence of real numbers such that all its convergent subsequences have the same limit $l$, then $\left(t_{k}\right)_{k}$ is convergent and $\lim _{k} t_{k}=l$.

Proof There are two subsequences $\left(t_{k_{n}}\right)_{n},\left(t_{k_{p}}\right)_{p}$ of $\left(t_{k}\right)_{k}$ such that

$$
\lim _{n} t_{k_{n}}=\liminf t_{k}, \quad \text { respectively, } \quad \lim _{p} t_{k_{p}}=\limsup t_{k} .
$$

Therefore, by hypothesis, we infer that $\liminf t_{k}=\lim \sup t_{k}=l$, that is, $\lim _{k} t_{k}=l$.

Lemma 3.2 Let $F: \mathbb{R}_{+} \rightarrow \mathbb{R}$ be an increasing map and $\left(t_{k}\right)_{k}$ be a sequence of positive real numbers. Then the following assertions hold:

(a) if $F\left(t_{k}\right) \underset{k}{\longrightarrow}-\infty$, then $t_{k} \underset{k}{\longrightarrow} 0$;

(b) if $\inf F=-\infty$ and $t_{k} \underset{k}{\longrightarrow} 0$, then $F\left(t_{k}\right) \underset{k}{\longrightarrow}-\infty$. 
Proof (a) First of all, we observe that $\left(t_{k}\right)_{k}$ is bounded. Indeed, if the sequence is unbounded above, one can find a subsequence $\left(t_{k_{p}}\right)_{p}$ such that $t_{k_{p}} \underset{p}{\longrightarrow} \infty$. Then, for every $\varepsilon>0$, there is $p_{\varepsilon} \in \mathbb{N}$ such that $t_{k_{p}} \geq \varepsilon$ for any $p \geq p_{\varepsilon}$. So $F(\varepsilon) \leq F\left(t_{k_{p}}\right)$, that is, $F(\varepsilon) \leq \lim _{p} F\left(t_{k_{p}}\right)=-\infty$, which is a contradiction.

Thereby $\left(t_{k}\right)_{k}$ is bounded, hence it has a convergent subsequence. Let $\left(t_{k_{n}}\right)_{n}$ be such a subsequence and $\alpha=\lim _{n} t_{k_{n}}$. Clearly $\alpha \geq 0$.

Suppose that $\alpha>0$ and choose $\varepsilon>0, \varepsilon<\alpha$. Then there exists $n_{\varepsilon} \in \mathbb{N}$ such that $t_{k_{n}} \in$ $(\alpha-\varepsilon, \alpha+\varepsilon)$ for all $n \geq n_{\varepsilon}$. As $F$ is increasing, we deduce that $F(\alpha-\varepsilon) \leq \lim _{n} F\left(t_{k_{n}}\right)=-\infty$ which contradicts $F(\alpha-\varepsilon) \in \mathbb{R}$.

Accordingly $\alpha=0$. Next we apply Lemma 3.1.

(b) Assume that $\inf F=-\infty$ and $t_{k} \underset{k}{\longrightarrow} 0$. Choose $\varepsilon>0$. There is $\alpha>0$ such that $F(\alpha)<$ $-\varepsilon$. Next, there exists $k_{\alpha} \in \mathbb{N}$ such that $t_{k}<\alpha$ for all $k \geq k_{\alpha}$. So, $F\left(t_{k}\right) \leq F(\alpha)<-\varepsilon$, for $k \geq k_{\alpha}$. Thus $F\left(t_{k}\right) \underset{k}{\longrightarrow}-\infty$.

Remark 3.1 According to Lemma 3.2, condition (F2) from Definition 3.1 may be replaced by

$\left(\mathrm{F} 2^{\prime}\right) \quad \inf F=-\infty$

or, also, by

$\left(\mathrm{F} 2^{\prime \prime}\right)$ there is $\left(t_{k}\right)_{k} \subset \mathbb{R}_{+}$such that $\lim _{k} F\left(t_{k}\right)=-\infty$.

By means of the previous remark, one can obtain new examples of functions of $\mathcal{F}$. In this respect, for $\alpha \in(0,1)$, we can consider $F_{1}(t)=-t^{-\alpha}, F_{2}(t)=\frac{t^{\alpha}}{1-e^{t}}$ and $F_{3}(t)=\frac{-1}{\arctan t^{\alpha}}$ (to check (F3) we take $\lambda \in(\alpha, 1)$ ).

Some properties of the set $\mathcal{F}$ are emphasized in the next proposition.

Proposition 3.1 Let consider $N \in \mathbb{N}, F_{1}, \ldots, F_{N} \in \mathcal{F}$ and $\alpha_{1}, \ldots, \alpha_{N} \in \mathbb{R}_{+}$. We define $F, G, H: \mathbb{R}_{+} \rightarrow \mathbb{R}$ by $F:=\min \left\{F_{1}, \ldots, F_{N}\right\}, G:=\max \left\{F_{1}, \ldots, F_{N}\right\}$ and $H:=\alpha_{1} F_{1}+\cdots+\alpha_{N} F_{N}$. Then $F, G, H \in \mathcal{F}$.

Proof If $\left(t_{k}\right)_{k} \subset \mathbb{R}_{+}$is such that $\lim _{k} F_{n}\left(t_{k}\right)=-\infty$ for some $n \in\{1, \ldots, N\}$, then $\lim _{k} F\left(t_{k}\right)=$ $-\infty$ and, also, $\lim _{k} H\left(t_{k}\right)=-\infty$. Next, since $\inf F_{n}=-\infty$ for all $1 \leq n \leq N$, we get $\inf G=-\infty$.

In order to verify (F3), we set $\lambda:=\max \left\{\lambda_{1}, \ldots, \lambda_{N}\right\}$, where $\lim _{t \backslash 0} t^{\lambda_{n}} F_{n}(t)=0,1 \leq n \leq N$. Then, for every $t \in(0,1)$, one has $t^{\lambda} \leq t^{\lambda_{n}}$ hence $t^{\lambda}|F(t)| \leq t^{\lambda_{n}}\left|F_{n}(t)\right|$ and $t^{\lambda}|H(t)| \leq$ $\alpha_{1} t^{\lambda}\left|F_{1}(t)\right|+\cdots+\alpha_{N} t^{\lambda}\left|F_{N}(t)\right|$ for all $t \in \mathbb{R}_{+}$and $n \in\{1, \ldots, N\}$. So, $\lim _{t \searrow 0} t^{\lambda} F(t)=0$ and $\lim _{t \searrow 0} t^{\lambda} H(t)=0$. Finally, as $\lim _{t \searrow_{0}} t^{\lambda}\left|F_{n}(t)\right| \leq \lim _{t \searrow_{0}} t^{\lambda_{n}}\left|F_{n}(t)\right|=0$ for every $n$, we deduce that $\lim _{t \backslash 0} t^{\lambda} G(t)=0$.

In the following remarks we provide a large subclass of $\mathcal{F}$.

Remark 3.2 Let $\mathcal{F}_{1}$ be the family of mappings $F: \mathbb{R}_{+} \rightarrow \mathbb{R}$ given by $F=G \circ f+g$, where $G \in \mathcal{F}$, and the functions $f: \mathbb{R}_{+} \rightarrow \mathbb{R}_{+}$and $g: \mathbb{R}_{+} \rightarrow \mathbb{R}$ satisfy the following conditions:

(a) $f$ is strictly increasing, $\inf f=0$ and there are $M, \delta \in \mathbb{R}_{+}$such that $t \leq M f(t)$ for all $t \in(0, \delta)$;

(b) $g$ is strictly increasing and there exists $\eta \in(0,1)$ such that $\lim _{t \searrow_{0}} t^{\eta} g(t)=0$.

Then $\mathcal{F}_{1} \subset \mathcal{F}$. 
Proof Choose $F \in \mathcal{F}_{1}$. Clearly, $F$ is strictly increasing. If $\left(t_{k}\right)_{k} \subset \mathbb{R}_{+}$is such that $\lim _{k} f\left(t_{k}\right)=$ 0 , then $\lim _{k} G\left(f\left(t_{k}\right)\right)=-\infty$ and so $\lim _{k} F\left(t_{k}\right)=-\infty$, this happens since the sequence $\left(g\left(t_{k}\right)\right)_{k}$ is bounded from above. Indeed, as $\lim _{k} f\left(t_{k}\right)=0$, there exists $k_{0}$ such that $\frac{t_{k}}{M} \leq f\left(t_{k}\right) \leq 1$, hence $t_{k} \leq M$, so $g\left(t_{k}\right) \leq g(M)$ for each $k>k_{0}$. According to Remark 3.1, it follows that $F$ satisfies (F2).

Next, let $\lambda_{1} \in(0,1)$ be such that $\lim _{s \backslash 0} s^{\lambda_{1}} G(s)=0$. Then, for every $\lambda \geq \max \left\{\eta, \lambda_{1}\right\}, \lambda<1$, one has

$$
\lim _{t \searrow 0}\left|t^{\lambda} F(t)\right| \leq \lim _{t \searrow 0}\left|\frac{t^{\lambda}}{(f(t))^{\lambda}}(f(t))^{\lambda} G(f(t))\right|+\lim _{t \searrow 0}\left|t^{\lambda} g(t)\right| \leq M^{\lambda} \lim _{s \searrow 0}\left|s^{\lambda} G(s)\right|=0 .
$$

Thereby (F3) is also verified.

Consequently, $F \in \mathcal{F}$.

Remark 3.3 Let $f: \mathbb{R}_{+} \rightarrow \mathbb{R}_{+}$and $g: \mathbb{R}_{+} \rightarrow \mathbb{R}$ be two maps satisfying the following conditions:

(a) $f$ is strictly increasing and $\inf f=0$;

(b) $g$ is strictly increasing and there exists $\eta \in(0,1)$ such that $\lim _{t \searrow_{0}} t^{\eta} g(t)=0$;

(c) there exists $\lambda_{1} \in(0,1)$ such that $\lim _{t \backslash 0} t^{\lambda_{1}} \ln f(t)=0$. In particular, this condition holds if $f$ is differentiable and there are $M, \delta \in \mathbb{R}_{+}$such that $t f^{\prime}(t) \leq M f(t)$ for every $t \in(0, \delta)$.

Then the function $F: \mathbb{R}_{+} \rightarrow \mathbb{R}, F(t):=\ln f(t)+g(t)$ belongs to $\mathcal{F}$.

Proof Conditions (F1) and (F2) can be checked as in Remark 3.2 taking into account that $G \in \mathcal{F}$, where $G(t)=\ln t$, and (F3) follows from the hypothesis (c).

In the particular case, when $f$ is differentiable, we use L'Hospital's rule and obtain

$$
\lim _{t \searrow 0} t^{\lambda} \ln f(t)=\frac{1}{\lambda} \lim _{t \searrow 0} \frac{f^{\prime}(t) t^{\lambda+1}}{f(t)} \leq \frac{M}{\lambda} \lim _{t \searrow 0} t^{\lambda}=0
$$

for every $\lambda \geq \max \left\{\eta, \lambda_{1}\right\}, \lambda<1$. Since $\lim _{t \searrow 0} t^{\lambda} g(t)=0$, we deduce $\lim _{t \searrow 0} t^{\lambda} F(t)=0$.

Some examples of functions $f$ which satisfy the requirement (a) from Remark 3.2 and the requirements (a) and (c) from Remark 3.3 are the following:

$$
\begin{aligned}
& f_{1}(t)=a t^{\alpha} \quad(0<\alpha<1), \quad f_{2}(t)=a \arctan t^{\alpha}, \\
& f_{3}(t)=a \ln \left(t^{\alpha}+1\right), \quad f_{4}(t)=a\left(b^{t}-1\right),
\end{aligned}
$$

for every $t \in \mathbb{R}_{+}$, where $a>0,0<\alpha \leq 1, b>1$. For Remark 3.3, we can further consider $f_{5}(t)=a_{n} t^{\alpha_{n}}+a_{n-1} t^{\alpha_{n-1}}+\cdots+a_{1} t^{\alpha_{1}}$, where $\alpha_{1}, \ldots, \alpha_{n} \in \mathbb{R}_{+}$and $a_{1}, \ldots, a_{n}$ are nonnegative numbers, $a_{n} \neq 0, n \in \mathbb{N}$. Indeed, as example for $f_{5}$, assuming that $\alpha_{1} \leq \alpha_{2} \leq \cdots \leq \alpha_{n}$, one has

$$
\begin{aligned}
\lim _{t \searrow 0} \frac{t f^{\prime}(t)}{f(t)} & =\lim _{t \searrow 0} \frac{t\left(\alpha_{n} a_{n} t^{\alpha_{n}-1}+\cdots+\alpha_{1} a_{1} t^{\alpha_{1}-1}\right)}{a_{n} t^{\alpha_{n}}+\cdots+a_{1} t^{\alpha_{1}}} \\
& =\lim _{t \searrow 0} \frac{\alpha_{n} a_{n} t^{\alpha_{n}-\alpha_{1}}+\cdots+\alpha_{2} a_{2} t^{\alpha_{2}-\alpha_{1}}+\alpha_{1} a_{1}}{a_{n} t^{\alpha_{n}-\alpha_{1}}+\cdots+a_{2} t^{\alpha_{2}-\alpha_{1}}+a_{1}}<\infty .
\end{aligned}
$$


The next theorem states a similar result as the Banach contraction principle for $F$ contractions.

Theorem 3.1 [11, Th. 2.1] Assume that $(X, \mathrm{~d})$ is a complete metric space, $F \in \mathcal{F}$ and $\omega$ : $X \rightarrow X$ is an F-contraction. Then $\omega$ has a unique fixed point e and for every $x \in X$, the sequence $\left(\omega^{p}(x)\right)_{p}$ converges to $e$.

The Banach contractions are particular cases of $F$-contractions, where $F(t)=\ln t$. In the following example, we show that there are $F$-contractions on a complete metric space which are not Banach contractions.

Example 3.1 Let $\left(\alpha_{m}\right)_{m \geq 1}$ be the sequence of real numbers given by $\alpha_{m}=m^{2}-\ln m$ and $X=[0,1] \cup\left\{\alpha_{m} ; m=1,2, \ldots\right\}$ endowed with the Euclidian metric $\mathrm{d}(x, y)=|x-y|$. Then $(X, \mathrm{~d})$ is a complete metric space. Consider the maps $F: \mathbb{R}_{+} \rightarrow \mathbb{R}, \omega: X \rightarrow X$ defined by $F(t)=\ln t^{\delta}+\beta t, \omega(x)=\eta x+\lambda$ if $x \in[0,1]$ and $\omega\left(\alpha_{m}\right)=\alpha_{m-1}$ for $m \geq 2$, where $\delta, \beta, \eta \in$ $\mathbb{R}_{+}, \eta<1, \lambda \in[0,1-\eta]$. Then $F \in \mathcal{F}$ and $\omega$ is an $F$-contraction which is not a Banach contraction, $e=\frac{\lambda}{1-\eta}$ being its fixed point.

Proof We first observe that $\alpha_{m+1}-\alpha_{m}=2 m+1-\ln \left(1+m^{-1}\right)>0$, hence $1 \leq \alpha_{m}<\alpha_{m+1}$ for all $m \geq 1$.

According to Remark 3.3, we deduce that $F \in \mathcal{F}$. We will prove that there is $\tau>0$ such that $\omega$ satisfies condition (1), that is,

$$
\frac{|\omega(x)-\omega(y)|^{\delta}}{|x-y|^{\delta}} e^{\beta|\omega(x)-\omega(y)|-\beta|x-y|} \leq e^{-\tau}, \quad \forall x, y \in X, \omega(x) \neq \omega(y) .
$$

Choose $x, y \in X$ such that $\omega(x) \neq \omega(y)$ and suppose, for instance, that $x<y$. Three cases can occur.

Case I: $x, y \in[0,1]$. Then

$$
\frac{|\omega(x)-\omega(y)|^{\delta}}{|x-y|^{\delta}} e^{\beta|\omega(x)-\omega(y)|-\beta|x-y|}=\frac{\eta^{\delta}(y-x)^{\delta}}{(y-x)^{\delta}} e^{\beta \eta(y-x)-\beta(y-x)}=\eta^{\delta} e^{\beta(y-x)(\eta-1)}<\eta^{\delta} \leq e^{-\tau}
$$

for every $\tau \in(0,-\delta \ln \eta)$.

Case II: $x=\alpha_{m}, y=\alpha_{m+p}, m \geq 2, p \geq 1$. Then

$$
\begin{aligned}
\frac{|\omega(x)-\omega(y)|^{\delta}}{|x-y|^{\delta}} e^{\beta|\omega(x)-\omega(y)|-\beta|x-y|} & =\frac{\left(\alpha_{m+p-1}-\alpha_{m-1}\right)^{\delta}}{\left(\alpha_{m+p}-\alpha_{m}\right)^{\delta}} e^{\beta\left(\alpha_{m+p-1}-\alpha_{m-1}-\alpha_{m+p}+\alpha_{m}\right)} \\
& =\frac{\left(2(m-1) p+p^{2}-\ln \left(1+\frac{p}{m-1}\right)\right)^{\delta}}{\left(2 m p+p^{2}-\ln \left(1+\frac{p}{m}\right)\right)^{\delta}} e^{\beta\left(-2 p-\ln \left(1+\frac{p}{m-1}\right)+\ln \left(1+\frac{p}{m}\right)\right)} \\
& <e^{-2 \beta p} \leq e^{-2 \beta} \leq e^{-\tau}
\end{aligned}
$$

for every $\tau \in(0,2 \beta]$.

Case III: $x \in[0,1], y=\alpha_{m}, m \geq 2$. Since

$$
\begin{aligned}
\left|\omega(x)-\omega_{m}\left(\alpha_{m}\right)\right|-\left|x-\alpha_{m}\right| & =\alpha_{m-1}-\eta x-\lambda-\alpha_{m}+x \\
& =-2 m+1+\ln \left(1+\frac{1}{m-1}\right)+(1-\eta) x-\lambda
\end{aligned}
$$




$$
\begin{aligned}
& <-2 m+1+\ln 2+1-\eta-\lambda \\
& \leq-2+\ln 2-\eta<0
\end{aligned}
$$

we get

$$
\frac{|\omega(x)-\omega(y)|^{\delta}}{|x-y|^{\delta}} e^{\beta|\omega(x)-\omega(y)|-\beta|x-y|}<e^{-\beta(2-\ln 2+\eta)} \leq e^{-\tau}
$$

for every $\tau \in(0, \beta(2-\ln 2+\eta)]$.

In conclusion, in any case, inequality (1) is fulfilled for every $\tau>0, \tau \leq \min \{-\delta \ln \eta, 2 \beta$, $\beta(2-\ln 2+\eta)\}$. More simple, since $1-\ln 2>0$, we can take $\tau \leq \min \{-\delta \ln \eta, \beta(\eta+1)\}$.

The mapping $\omega$ is not a Banach contraction because

$$
\lim _{m} \frac{\left|\omega\left(\alpha_{m+1}\right)-\omega\left(\alpha_{m}\right)\right|}{\left|\alpha_{m+1}-\alpha_{m}\right|}=\lim _{m} \frac{2 m+1-\ln \left(1+\frac{1}{m}\right)}{2 m-1-\ln \left(1+\frac{1}{m-1}\right)}=1 .
$$

Theorem 3.2 Let $F \in \mathcal{F}$ be continuous and suppose that $(X, \mathrm{~d})$ is complete. For every $n \geq 1$, let $\omega_{n}: X \rightarrow X$ be an F-contraction and $e_{n}$ be its fixed point. Assume that:

(a1) the sequence $\left(\omega_{n}\right)_{n}$ converges pointwise to a map $\omega: X \rightarrow X$,

(a2) $\inf _{n} \tau_{n}>0$, where $\tau_{n}$ is a constant associated with $\omega_{n}$ from (1).

Then $\omega$ is an F-contraction. If we further have

(a3) $\left(e_{n}\right)_{n}$ is convergent and $e=\lim _{n} e_{n}$,

then $\omega(e)=e$.

Proof Set $\tau=\inf _{n} \tau_{n}$. Then

$$
\tau+F\left(\mathrm{~d}\left(\omega_{n}(x), \omega_{n}(y)\right)\right) \leq F(\mathrm{~d}(x, y)), \quad \forall n \geq 1, \forall x, y \in X, \omega_{n}(x) \neq \omega_{n}(y)
$$

Let $x, y \in X$ be such that $\omega(x) \neq \omega(y)$. Since $\lim _{n} \mathrm{~d}\left(\omega_{n}(x), \omega_{n}(y)\right)=\mathrm{d}(\omega(x), \omega(y))>0$ for every $\delta>0, \delta<\mathrm{d}(\omega(x), \omega(y))$, one can find $N_{1} \in \mathbb{N}$ such that

$$
\mathrm{d}(\omega(x), \omega(y))-\delta<\mathrm{d}\left(\omega_{n}(x), \omega_{n}(y)\right)<\mathrm{d}(\omega(x), \omega(y))+\delta, \quad \forall n \geq N_{1}
$$

Choose $\varepsilon>0$. By the pointwise convergence of $\left(\omega_{n}\right)_{n}$, there exists $N_{2} \in \mathbb{N}$ such that

$$
\mathrm{d}\left(\omega_{n}(x), \omega(x)\right)<\frac{\varepsilon}{2} \quad \text { and } \quad \mathrm{d}\left(\omega_{n}(y), \omega(y)\right)<\frac{\varepsilon}{2}, \quad \forall n \geq N_{2} .
$$

Let $N=\max \left\{N_{1}, N_{2}\right\}$. We have

$$
\mathrm{d}(\omega(x), \omega(y)) \leq \mathrm{d}\left(\omega(x), \omega_{n}(x)\right)+\mathrm{d}\left(\omega_{n}(x), \omega_{n}(y)\right)+\mathrm{d}\left(\omega_{n}(y), \omega(y)\right)<\varepsilon+\mathrm{d}\left(\omega_{n}(x), \omega_{n}(y)\right)
$$

for any $n \geq N$. Hence, for each $n \geq N$, we get by (3)

$$
\begin{aligned}
\tau+F(\mathrm{~d}(\omega(x), \omega(y))) & \leq \tau+F\left(\varepsilon+\mathrm{d}\left(\omega_{n}(x), \omega_{n}(y)\right)\right) \\
& \leq F(\mathrm{~d}(x, y))+F\left(\varepsilon+\mathrm{d}\left(\omega_{n}(x), \omega_{n}(y)\right)\right)-F\left(\mathrm{~d}\left(\omega_{n}(x), \omega_{n}(y)\right)\right) \\
& \leq F(\mathrm{~d}(x, y))+F(\varepsilon+\delta+\mathrm{d}(\omega(x), \omega(y)))-F(\mathrm{~d}(\omega(x), \omega(y))-\delta),
\end{aligned}
$$

where at the last inequality we used (4). 
Letting $\varepsilon \searrow 0$ and $\delta \searrow 0$ it follows, using the continuity of $F$,

$$
\tau+F(\mathrm{~d}(\omega(x), \omega(y))) \leq F(\mathrm{~d}(x, y))
$$

so $\omega$ is an $F$-contraction.

The last assertion of statement comes taking into consideration that each $\omega_{n}$ is contractive as follows:

$$
\begin{aligned}
\mathrm{d}(e, \omega(e)) & \leq \mathrm{d}\left(e, \omega_{n}\left(e_{n}\right)\right)+\mathrm{d}\left(\omega_{n}\left(e_{n}\right), \omega_{n}(e)\right)+\mathrm{d}\left(\omega_{n}(e), \omega(e)\right) \\
& \leq \mathrm{d}\left(e, e_{n}\right)+\mathrm{d}\left(e_{n}, e\right)+\mathrm{d}\left(\omega_{n}(e), \omega(e)\right) \underset{n}{\longrightarrow} 0 .
\end{aligned}
$$

A property concerning the compositions of $F$-contractions which will be used in the next section is given in the following.

Proposition 3.2 If $\left(\omega_{k}\right)_{k=1}^{K}$ is a collection of F-contractions on $X$ to itself, then the map $\omega:=\omega_{1} \circ \cdots \circ \omega_{K}$ is a F-contraction.

Proof Set $\tau=\min \left\{\tau_{1}, \ldots, \tau_{K}\right\}$, where $\tau_{k}$ is the constant from (1) associated to $\omega_{k}, k=$ $1, \ldots, K$. We consider $K=2$. For the general case, one can proceed inductively.

$$
\begin{aligned}
\tau+F\left(\mathrm{~d}\left(\left(\omega_{1} \circ \omega_{2}\right)(x),\left(\omega_{1} \circ \omega_{2}\right)(y)\right)\right) & \leq \tau_{2}+\tau_{1}+F\left(\mathrm{~d}\left(\left(\omega_{1} \circ \omega_{2}\right)(x),\left(\omega_{1} \circ \omega_{2}\right)(y)\right)\right) \\
& \leq \tau_{2}+F\left(\mathrm{~d}\left(\omega_{2}(x), \omega_{2}(y)\right)\right) \leq F(\mathrm{~d}(x, y))
\end{aligned}
$$

for all $x, y \in X, \omega_{1}\left(\omega_{2}(x)\right) \neq \omega_{1}\left(\omega_{2}(y)\right)$.

\section{Application: iterated function systems}

We assume that $(X, \mathrm{~d})$ is a complete metric space.

The classical iterated function system (IFS) introduced by Hutchinson [1] consists of a finite family of Banach contractions on $X$ to itself. There is a unique nonempty compact subset of $X$ invariant with respect to these contractions. In what follows, we extend this IFS by considering a family of $F$-contractions.

Definition 4.1 For each $k=1, \ldots, K$, let $F_{k} \in \mathcal{F}$ and $\omega_{k}: X \rightarrow X$ be an $F_{k}$-contraction. The family $\left(\omega_{k}\right)_{k=1}^{K}$ is called an iterated function system, abbreviated IFS. The set function $\mathcal{S}$ : $\mathcal{K}(X) \rightarrow \mathcal{K}(X)$ defined by $\mathcal{S}(B)=\bigcup_{k=1}^{K} \omega_{k}(B)$ is called the associated Hutchinson operator. A set $A \in \mathcal{K}(X)$ is said to be an attractor of the IFS whenever $\mathcal{S}(A)=A$.

We will prove that in a certain condition relating to the mappings $F_{1}, \ldots, F_{K}$, such an IFS has a unique attractor. We first need the following lemma.

Lemma 4.1 Let $\omega: X \rightarrow X$ bean F-contraction, where $F \in \mathcal{F}$. Then the mapping $A \mapsto \omega(A)$ is an $F$-contraction too from $\mathcal{K}(X)$ into itself.

Proof Choose $A, B \in \mathcal{K}(X)$ such that $h(\omega(A), \omega(B))>0$. Assume that

$$
h(\omega(A), \omega(B))=\mathrm{D}(\omega(A), \omega(B))=\sup _{x \in A} \inf _{y \in B} \mathrm{~d}(\omega(x), \omega(y))>0 .
$$


By hypothesis, there is $\tau>0$ such that $\tau+F(\mathrm{~d}(\omega(x), \omega(y))) \leq F(\mathrm{~d}(x, y))$ for every $x, y \in X$, $\omega(x) \neq \omega(y)$.

Using (5), the compactness of $A$ and the continuity of $\omega$, one can find $a \in A$ such that $\mathrm{D}(\omega(A), \omega(B))=\inf _{y \in B} \mathrm{~d}(\omega(a), \omega(y))>0$, so $\mathrm{d}(\omega(a), \omega(y))>0$ for all $y \in B$. Therefore

$$
\tau+F\left(\inf _{y \in B} \mathrm{~d}(\omega(a), \omega(y))\right) \leq \tau+F(\mathrm{~d}(\omega(a), \omega(y))) \leq F(\mathrm{~d}(a, y)), \quad \forall y \in B
$$

that is,

$$
\tau+F(h(\omega(A), \omega(B))) \leq F(\mathrm{~d}(a, y)), \quad \forall y \in B
$$

Let $b \in B$ be such that $\mathrm{d}(a, b)=\inf _{y \in B} \mathrm{~d}(a, y)$. Then, by (6), we get

$$
\begin{aligned}
\tau+F(h(\omega(A), \omega(B))) & \leq F(\mathrm{~d}(a, b))=F\left(\inf _{y \in B} \mathrm{~d}(a, y)\right) \\
& \leq F\left(\sup _{x \in A} \inf _{y \in B} \mathrm{~d}(x, y)\right)=F(\mathrm{D}(A, B)) \leq F(h(A, B)) .
\end{aligned}
$$

Consequently, $\tau+F(h(\omega(A), \omega(B))) \leq F(h(A, B))$, as required.

Theorem 4.1 We consider $K \in \mathbb{N}, F_{1}, \ldots, F_{K} \in \mathcal{F}$ and define $F=\max _{1 \leq k \leq K} F_{k}$. Assume that the map $g_{k}:=F-F_{k}$ is nondecreasing for all $1 \leq k \leq K$. For each $k=1, \ldots, K$, let $\omega_{k}: X \rightarrow X$ be an $F_{k}$-contraction. Then $\mathcal{S}$ is an F-contraction and the IFS $\left(\omega_{k}\right)_{k=1}^{K}$ has a unique attractor which is successively approximated in the Hausdorff-Pompeiu metric by $\left(\mathcal{S}^{p}(B)\right)_{p}$ for every $B \in \mathcal{K}(X)$.

Proof First of all, notice that $F \in \mathcal{F}$ according to Proposition 3.1.

By hypothesis, there are $\tau_{1}, \ldots, \tau_{K} \in \mathbb{R}_{+}$such that

$$
\tau_{k}+F_{k}\left(\mathrm{~d}\left(\omega_{k}(x), \omega_{k}(y)\right)\right) \leq F_{k}(\mathrm{~d}(x, y)), \quad \forall x, y \in X, \omega_{k}(x) \neq \omega_{k}(y), k \in\{1, \ldots, K\} .
$$

Set $\tau=\min \left\{\tau_{1}, \ldots, \tau_{K}\right\}>0$.

Let $A, B \in \mathcal{K}(X)$ be such that $h(\mathcal{S}(A), \mathcal{S}(B))>0$. By Lemma 2.2, we get

$$
0<h(\mathcal{S}(A), \mathcal{S}(B)) \leq \sup _{1 \leq k \leq K} h\left(\omega_{k}(A), \omega_{k}(B)\right)=h\left(\omega_{k_{0}}(A), \omega_{k_{0}}(B)\right)
$$

for some $k_{0} \in\{1, \ldots, K\}$. Using now Lemma 4.1 and the hypotheses, one obtains

$$
\begin{aligned}
\tau+F(h(\mathcal{S}(A), \mathcal{S}(B))) & \leq \tau+F\left(h\left(\omega_{k_{0}}(A), \omega_{k_{0}}(B)\right)\right) \\
& \leq \tau_{k_{0}}+F_{k_{0}}\left(h\left(\omega_{k_{0}}(A), \omega_{k_{0}}(B)\right)\right)+g_{k_{0}}\left(h\left(\omega_{k_{0}}(A), \omega_{k_{0}}(B)\right)\right) \\
& \leq F_{k_{0}}(h(A, B))+g_{k_{0}}(h(A, B))=F(h(A, B)),
\end{aligned}
$$

which assures that $\mathcal{S}$ is an $F$-contraction.

The rest of assertions from the statement now follow by applying Theorem 3.1, the metric space $(\mathcal{K}(X), h)$ being complete. 
Remark 4.1 If there exists a map $F \in \mathcal{F}$ such that $\omega_{k}$ is an $F$-contraction for all $1 \leq k \leq K$, then the IFS $\left(\omega_{k}\right)_{k=1}^{K}$ has a unique attractor which is successively approximated by $\left(\mathcal{S}^{p}(B)\right)_{p}$ for every $B \in \mathcal{K}(X)$.

Lemma 4.2 Let $\left(\omega_{n}\right)_{n}$ be a sequence of contractive self-mappings on $X$ pointwise convergent to $\omega: X \rightarrow X$. Then $\omega_{n}(B) \underset{n}{\longrightarrow} \omega(B)$ for every $B \in \mathcal{K}(X)$, the converging process being taken with respect to the Hausdorf-Pompeiu metric.

Proof Choose $B \in \mathcal{K}(X)$. We have to show that $h\left(\omega_{n}(B), \omega(B)\right) \underset{n}{\longrightarrow} 0$. For this purpose, we suppose by contradiction that there is $\varepsilon_{0}>0$ such that $h\left(\omega_{n}(B), \omega(B)\right) \geq \varepsilon_{0}$ for infinitely many integers $n \geq 1$. Two cases can occur.

Case I: There exists a sequence of positive integers $\left(n_{p}\right)_{p}$ such that

$$
\mathrm{D}\left(\omega_{n_{p}}(B), \omega(B)\right)=\sup _{x \in B} \inf _{y \in B} \mathrm{~d}\left(\omega_{n_{p}}(x), \omega(y)\right) \geq \varepsilon_{0}, \quad \forall p \in \mathbb{N} .
$$

So, for every $p \in \mathbb{N}$, one can find $x_{p} \in B$ such that $\inf _{y \in B} \mathrm{~d}\left(\omega_{n_{p}}\left(x_{p}\right), \omega(y)\right) \geq \varepsilon_{0}$, that is,

$$
\mathrm{d}\left(\omega_{n_{p}}\left(x_{p}\right), \omega(y)\right) \geq \varepsilon_{0}, \quad \forall y \in B, p \geq 1 .
$$

Since $B$ is compact, the sequence $\left(x_{p}\right)_{p}$ admits a convergent subsequence which, for simplicity, will be denoted in the same way. Thus $x_{p} \rightarrow y \in B$. Now, using the hypotheses and (8), we get

$$
\begin{aligned}
\varepsilon_{0} & \leq \mathrm{d}\left(\omega_{n_{p}}\left(x_{p}\right), \omega(y)\right) \leq \mathrm{d}\left(\omega_{n_{p}}\left(x_{p}\right), \omega_{n_{p}}(y)\right)+\mathrm{d}\left(\omega_{n_{p}}(y), \omega(y)\right) \\
& \leq \mathrm{d}\left(x_{p}, y\right)+\mathrm{d}\left(\omega_{n_{p}}(y), \omega(y)\right) \underset{p}{\longrightarrow} 0,
\end{aligned}
$$

which is a contradiction.

Case II: The case when there is $\left(n_{p}\right)_{p} \subset \mathbb{N}$ such that $\mathrm{D}\left(\omega(B), \omega_{n_{p}}(B)\right) \geq \varepsilon_{0}$ for every $p \geq 1$, can be treated analogously.

Accordingly, $\omega(B)=\lim _{n} \omega_{n}(B)$.

Theorem 4.2 Let $K \in \mathbb{N}$ be given. For every $n \geq 1$ and $k \in\{1, \ldots, K\}$, we consider the mappings $F_{k} \in \mathcal{F}$ and $\omega_{k}^{n}: X \rightarrow X$. Assume that the following conditions are fulfilled:

(C1) for each $k=1, \ldots, K, F_{k}$ is continuous and the map $g_{k}: \mathbb{R}_{+} \rightarrow[0, \infty), g_{k}=F-F_{k}$, where $F=\max \left\{F_{1}, \ldots, F_{K}\right\}$, is nondecreasing;

(C2) for each $n \in \mathbb{N}$ and $1 \leq k \leq K, \omega_{k}^{n}$ is an $F_{k}$-contraction and $\inf _{n} \tau_{k}^{n}>0$, where $\tau_{k}^{n}$ is a constant associated with $\omega_{k}^{n}$ from (1);

(C3) the sequence $\left(\omega_{k}^{n}\right)_{n}$ converges pointwise to a map $\omega_{k}: X \rightarrow X$ for every $k=1, \ldots, K$;

(C4) if, for every $n \geq 1, A_{n}$ is the attractor of IFS $\left(\omega_{k}^{n}\right)_{k=1}^{K}$, then the sequence $\left(A_{n}\right)_{n}$ converges in the space $(\mathcal{K}(X), h)$ to a set $A$.

Then $\left(\omega_{k}\right)_{k=1}^{K}$ is an IFS which can be regarded as the limit of the sequence of IFSs $\left(\left(\omega_{k}^{n}\right)_{k=1}^{K}\right)_{n}$. Moreover, $A$ is the attractor of the respective IFS.

Proof Using (C2) and (C3), we deduce from Theorem 3.2 that $\left(\omega_{k}\right)_{k=1}^{K}$ is an IFS. According to Theorem 4.1, the Hutchinson operator $\mathcal{S}_{n}$ associated with IFS $\left(\omega_{k}^{n}\right)_{k=1}^{K}$ is an Fcontraction in the complete metric space $(\mathcal{K}(X), h)$, so it has a unique set-'fixed point' $A_{n}$ for all $n=1,2, \ldots$. 
In order to apply again Theorem 3.2 , we notice that $\mathcal{S}_{n}(B) \underset{n}{\longrightarrow} \mathcal{S}(B)$ with respect to the Hausdorff-Pompeiu metric for every $B \in \mathcal{K}(X)$. Indeed, by means of Lemmas 2.2 and 4.2, one has

$$
\lim _{n} h\left(\mathcal{S}_{n}(B), \mathcal{S}(B)\right) \leq \lim _{n} \max _{1 \leq k \leq K} h\left(\omega_{k}^{n}(B), \omega(B)\right)=0
$$

Next, condition (a2) follows from (C2), via the proof of Theorem 4.1, which assures that one of the constants associated with $\mathcal{S}_{n}$ from $(1)$ is $\min \left\{\tau_{1}^{n}, \tau_{2}^{n}, \ldots, \tau_{K}^{n}\right\}$. Condition (C4) implies (a3).

The conclusion of Theorem 3.2 says that $\mathcal{S}(A)=A$, i.e., $A$ is the attractor of the IFS $\left(\omega_{k}\right)_{k=1}^{K}$.

Example 4.1 We are in the settings from Example 3.1. Let $K \in \mathbb{N}, K \geq 2$. Consider $\delta, \beta_{1}, \ldots, \beta_{K} \in \mathbb{R}_{+}$and, for every $k=1, \ldots, K, n \in \mathbb{N}, t \in \mathbb{R}_{+}$and $x \in X$, let define $F_{k}(t)=$ $\ln t^{\delta}+\beta_{k} t, \omega_{k}^{n}(x)=\eta_{n} x+\lambda_{k}$, where $\eta_{n}=\frac{n}{(n+1)(K+1)}, \lambda_{k}=\frac{K(k-1)}{K^{2}-1}$ if $x \in[0,1]$ and $\omega_{k}^{n}\left(\alpha_{m}\right)=\alpha_{m-1}$ for $m \geq 2$. Then the hypotheses of Theorem 4.2 are fulfilled.

Proof From Example 3.1, it follows that $F_{k} \in \mathcal{F}$ and $\omega_{k}^{n}$ is an $F_{k}$-contraction for each $k=$ $1, \ldots, K$ and $n \geq 1$. Hence $\left(\omega_{k}^{n}\right)_{k=1}^{K}$ is an IFS and, according to Theorem 4.1, the function $F-F_{k}$ being nondecreasing, we deduce that it has a unique attractor $A_{n}$ for every $n \geq 1$. Notice that all the mappings $\omega_{k}^{n}(n \geq 1,1 \leq k \leq K)$ are $F$-contractions.

Clearly, $F_{k}, 1 \leq k \leq K$, are continuous hence $(\mathrm{C} 1)$ is satisfied.

Next, as $\eta_{n} \in\left[\frac{1}{2(K+1)}, \frac{1}{K+1}\right)$, we get

$$
-\delta \ln \eta_{n}>\delta \ln (K+1) \quad \text { and } \quad \beta_{k}\left(\eta_{n}+1\right) \geq \min _{1 \leq k \leq K} \beta_{k}\left(\frac{1}{2(K+1)}+1\right), \quad \forall k, n
$$

So, for every $k=1, \ldots, K$ and $n \geq 1$, one can find $\tau_{k}^{n} \in\left(0, \min \left\{-\delta \ln \eta_{n}, \beta_{k}\left(\eta_{n}+1\right)\right\}\right]$ such that $\inf _{n} \tau_{k}^{n}>0$. Therefore (C2) holds.

The pointwise convergence of the sequence $\left(\omega_{k}^{n}\right)_{n}$ to $\omega_{k}:=\frac{1}{K+1} x+\lambda_{k}$, for every $k=$ $1, \ldots, K$, is obvious, hence $(\mathrm{C} 3)$ is verified.

It remains to show that also condition (C4) is satisfied. We claim that, for every $n \in \mathbb{N}$, $A_{n} \subset[0,1]$. Indeed, using Proposition 3.2, it follows that for each $p \geq 1$, the map $\omega_{i_{1} i_{2} \ldots i_{p}}^{n}$ is an $F$-contraction, where we denoted $\omega_{i_{1} \ldots i_{p}}^{n}:=\omega_{i_{1}}^{n} \circ \cdots \circ \omega_{i_{p}}^{n}$ for $i_{1}, \ldots, i_{p} \in\{1, \ldots, K\}$. It is easy to check that $A_{n}$ is also an attractor for the IFS $\left(\omega_{i_{1} \ldots i_{p}}^{n}\right)_{i_{1}, \ldots, i_{p}=1}^{K}$. Next, since $A_{n}$ is compact, there is $M \in \mathbb{N}$ such that $A_{n} \subset[0,1] \cup\left\{\alpha_{1}, \ldots, \alpha_{M}\right\}$. Obviously, if $p \geq M$, then $\omega_{i_{1} \ldots i_{p}}^{n}\left(\alpha_{m}\right) \leq 1$ for every $m=1, \ldots, M$, hence

$$
A_{n} \subset \bigcup_{i_{1}, \ldots, i_{p}=1}^{K} \omega_{i_{1} \ldots i_{p}}^{n}\left(A_{n}\right) \subset[0,1]
$$

Now, we intend to prove that $A_{n} \subset A_{n+1}$ for each $n \geq 1$. Set $B=[0,1] \in \mathcal{K}(X)$ and choose $n \in \mathbb{N}$. Since $\eta_{n}<\eta_{n+1}$, it follows

$$
\omega_{k}^{n}(B)=\left[\lambda_{k}, \eta_{n}+\lambda_{k}\right] \subset\left[\lambda_{k}, \eta_{n+1}+\lambda_{k}\right]=\omega_{k}^{n+1}(B), \quad \forall 1 \leq k \leq K .
$$


Then, inductively, we obtain $\omega_{i_{1} \ldots i_{p}}^{n}(B) \subset \omega_{i_{1} \ldots i_{p}}^{n+1}(B)$ for every $p \geq 1,1 \leq i_{1}, \ldots, i_{p} \leq K$. Therefore

$$
\mathcal{S}_{n}^{p}(B)=\bigcup_{i_{1}, \ldots, i_{p}=1}^{K} \omega_{i_{1} \ldots i_{p}}^{n}(B) \subset \bigcup_{i_{1}, \ldots, i_{p}=1}^{K} \omega_{i_{1} \ldots i_{p}}^{n+1}(B)=\mathcal{S}_{n+1}^{p}(B) .
$$

By Theorem 4.1, one has $\mathcal{S}_{n}^{p}(B) \underset{p}{\longrightarrow} A_{n}$ and $\mathcal{S}_{n+1}^{p}(B) \underset{p}{\longrightarrow} A_{n+1}$. Then, using Lemma 2.1 and (10), we get

$$
\begin{aligned}
\mathrm{D}\left(A_{n}, A_{n+1}\right) & \leq \mathrm{D}\left(A_{n}, \mathcal{S}_{n}^{p}(B)\right)+\mathrm{D}\left(\mathcal{S}_{n}^{p}(B), \mathcal{S}_{n+1}^{p}(B)\right)+\mathrm{D}\left(\mathcal{S}_{n+1}^{p}(B), A_{n+1}\right) \\
& =\mathrm{D}\left(A_{n}, \mathcal{S}_{n}^{p}(B)\right)+\mathrm{D}\left(\mathcal{S}_{n+1}^{p}(B), A_{n+1}\right) \underset{p}{\longrightarrow} 0 .
\end{aligned}
$$

Hence $\mathrm{D}\left(A_{n}, A_{n+1}\right)=0$ so, applying again Lemma 2.1, $A_{n} \subset A_{n+1}$.

The convergence of $\left(A_{n}\right)_{n}$ now follows from Lemma 2.3 taking into consideration that, by (9), we have

$$
\lim _{n} A_{n}=\overline{\bigcup_{n} A_{n}} \subset[0,1]
$$

Thus condition (P4) is verified.

Remark 4.2 In the previous example, we can also take:

$1^{o} \quad F_{k}(t)=\ln t^{\delta_{k}}+\beta t, \delta_{1}, \ldots, \delta_{K}, \beta \in \mathbb{R}_{+}$

and/or

$2^{o} \omega_{k}^{n}(x)=\eta_{n} x+\lambda_{k}, \eta_{n}=\frac{n+1}{2 n(K+1)}, \lambda_{k}=\frac{K(k-1)}{K^{2}-1}$ if $x \in[0,1]$ for every $n \geq 1,1 \leq k \leq K$. In this event, the sequence of attractors $\left(A_{n}\right)_{n}$ is descending and, according to Lemma 2.3, $\lim _{n} A_{n}=\bigcap_{n} A_{n}$.

\section{Competing interests}

The author declares that they have no competing interests.

\section{Acknowledgements}

The author thanks the referees for their valuable comments and useful suggestions in improving the paper.

Received: 13 June 2013 Accepted: 5 September 2013 Published: 08 Nov 2013

\section{References}

1. Hutchinson, J: Fractals and self-similarity. Indiana Univ. Math. J. 30, 713-747 (1981)

2. Barnsley, MF: Fractals Everywhere, 2nd edn. Academic Press, Boston (1993)

3. Mihail, A, Miculescu, R: Applications of fixed point theorems in the theory of generalized IFS. Fixed Point Theory Appl. 2008, Article ID 312876 (2008). doi:10.1155/2008/312876

4. Mihail, A, Miculescu, R: Generalized IFSs on noncompact spaces. Fixed Point Theory Appl. 2010, Article ID 584215 (2010). doi:10.1155/2010/584215

5. Dumitru, D: Generalized iterated function systems containing Meir-Keeler functions. An. Univ. Bucureşti, Math. LVIII 3-15 (2009)

6. Strobin, F, Swaczyna, J: On a certain generalization of the iterated function system. Bull. Aust. Math. Soc. 87(1), 37-54 (2013)

7. Secelean, NA: The existence of the attractor of countable iterated function systems. Mediterr. J. Math. 9(1), 61-79 (2012)

8. Llorens-Fuster, E, Petruşel, A, Yao, J-C: Iterated function systems and well-possedness. Chaos Solitons Fractals 41 , 1561-1568 (2009) 
9. Petruşel, A: Dynamical systems, fixed points and fractals. Pure Math. Appl. 13(1-2), 275-281 (2002)

10. Berinde, V: Iterative Approximation of Fixed Points. Lecture Notes in Mathematics. Springer, Berlin (2006)

11. Wardowski, D: Fixed points of a new type of contractive mappings in complete metric spaces. Fixed Point Theory Appl. 87, 2012/1/94 (2012). doi:10.1186/1687-1812-2012-94

12. Secelean, NA: Countable Iterated Function Systems. Lambert Academic Publishing, Colne (2013)

10.1186/1687-1812-2013-277

Cite this article as: Secelean: Iterated function systems consisting of F-contractions. Fixed Point Theory and Applications 2013, 2013:277

Submit your manuscript to a SpringerOpen ${ }^{\circ}$ journal and benefit from:

- Convenient online submission

- Rigorous peer review

- Immediate publication on acceptance

Open access: articles freely available online

- High visibility within the field

- Retaining the copyright to your article 